\title{
NOUVELLE
}

\section{La voie Notch \\ au centre du mécanisme \\ de leucémogenèse dans \\ un modèle murin de leucémies T}

Philippe Kastner, Susan Chan
Institut de Génétique et de Biologie Moléculaire et Cellulaire, 1, rue Laurent Fries, BP 10142, 67404 IIIkirch Cedex, France. scpk@igbmc.u-strasbg.fr
> Le traitement des leucémies aiguës lymphoblastiques $T$ ( $L A L-T$ ) repose principalement sur des protocoles de chimiothérapie et de radiothérapie, lourds à supporter, et qui échouent pour environ $25 \%$ des patients. Tout comme pour la leucémie myéloïde chronique (traitée avec l'Imatimib, inhibiteur de tyrosine kinase ciblé sur bcr-abl), la définition d'une thérapie ciblée sur les voies moléculaires impliquées dans la transformation des LAL-T pourrait améliorer ces traitements. La voie de signalisation en aval des récepteurs Notch, qui est spontanément activée dans les cellules leucémiques de près de $50 \%$ des patients atteints de LAL-T [1], est à cet égard une cible particulièrement prometteuse. Les récepteurs membranaires Notch (il en existe 4, mais c'est surtout de Notchl qu'il s'agit ici), après interaction avec des ligands présents sur d'autres cellules, subissent une cascade de clivages protéolytiques, notamment par la $\gamma$-sécrétase, qui libère leur partie intracellulaire qui agit ensuite comme cofac$(\rightarrow) \mathrm{m} / \mathrm{s}$ 2006, n० 8-9, P. 685 teur transcriptionnel $(\rightarrow)$.

Chez les patients atteints de LAL-T, I'activation de Notch est souvent associée à des mutations du gène Notchl qui augmentent sa sensibilité au clivage par la $\gamma$-sécrétase (mutations du domaine d'hétérodimérisation), ou qui entraînent des troncations dans la partie carboxyterminale augmentant l'activité de cette protéine [1]. Ainsi l'utilisation d'inhibiteurs de $\gamma$-sécrétase (IGS) est envisagée comme approche thérapeutique pour ces leucémies. Plusieurs questions restent cependant sans réponse : les IGS sont-ils efficaces comme moyen thérapeutique? Quels sont les mécanismes qui conduisent à l'activation de Notchl ? Lesquelles parmi les cibles de Notchl sont responsables de ses propriétés oncogéniques? La caractérisation par notre équipe d'un nouveau modèle murin de leucémies $T$ dépendantes de Notch (souris $I k^{L / L}$ ) [2] permettra d'aborder ces questions dans un système facilement manipulable.

Les souris $I k^{L / L}$ portent une mutation hypomorphe dans le gène codant pour Ikaros [3], un répresseur transcriptionnel de la famille des protéines à doigts de zinc [4]. Plusieurs études ont impliqué Ikaros comme un gène suppresseur de tumeurs pour les leucémies/lymphomes T. Ainsi, des pertes alléliques, ou des mutations ponctuelles conférant à Ikaros une action dominante négative sont fréquemment détectées dans des lymphomes thymiques murins induits par des agents mutagènes ou par irradiation [5-7]. De même, toutes les souris $I k^{L / L}$,

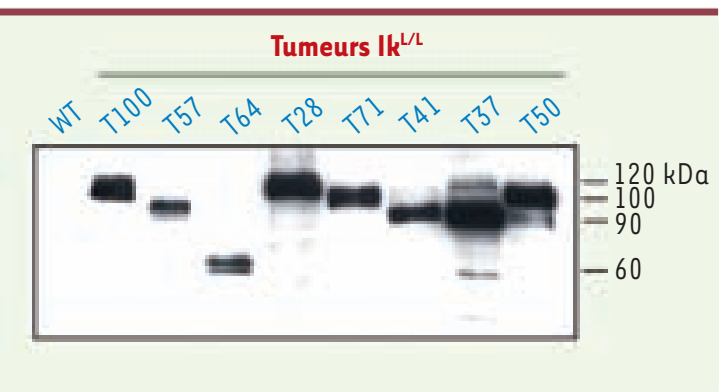

Figure 1. Formes clivées de Notchl dans les tumeurs $I k^{L / L}$. Analyse par Western blot d'extraits nucléaires de cellules provenant de thymocytes WT ou de tumeurs $I k^{L / L}$. Remarquer l'abondance des protéines clivées dans les tumeurs et la variabilité de taille de celles-ci. comme celles dont le gène contient des mutations de type «dominant-négatif » d'lkaros, développent des lymphomes thymiques $[2,8,9]$. Chez les souris $I k^{L / L}$, les tumeurs émergentes sont détectées dans le thymus entre l'âge de 2 et 3 mois, évoluent souvent en leucémie, et tuent toutes les souris avant l'âge de 6 mois. La constance de ce phénotype fait donc de cette lignée un outil de choix pour appréhender les mécanismes impliqués dans la transformation des thymocytes.

La voie Notch est spécifiquement activée dans les tumeurs $I k^{L / L}$

Grâce à une analyse du profil transcriptionnel, nous avons observé que plusieurs gènes cibles de la voie Notch (Notch1, Hes-1, Deltex-1, pT $\alpha$ ) étaient toujours fortement exprimés dans les tumeurs $\mathrm{I} \mathrm{K}^{\mathrm{L} / \mathrm{L}}$, indiquant l'activation de cette voie. L'activation récurrente de la voie Notch dans les tumeurs $I k^{L / L}$ a été confirmée par la présence abondante de formes clivées nucléaires de Notchl (Figure 1). Cette activation est de plus un événement précoce, puisqu'on la détecte déjà dans les tumeurs émergentes. L'analyse des formes nucléaires de Notchl dans les tumeurs $I k^{L / L}$ a par ailleurs révélé la présence fréquente (environ $70 \%$ des cas) de formes clivées plus petites que la forme attendue de 120 kDa (Figure I). Ces formes tronquées résultent de 
mutations ponctuelles dans les séquences codant la région carboxy-terminale, induisant un décalage de phase conduisant à la délétion du domaine PEST (impliqué dans la stabilité de Notchl). De façon intéressante, ces mutations sont similaires à certaines de celles décrites dans les LAL-T humaines (Figure 2). Par ailleurs, comme c'est le cas pour les LAL-T, l'activation de la voie Notch joue un rôle essentiel dans la prolifération des cellules tumorales $\mathrm{Ik}^{\mathrm{L} / \mathrm{L}}$. En effet, la prolifération de lignées cellulaires établies à partir de tumeurs primaires est inhibée par des IGS, et restaurée si la forme clivée de Notchl est exprimée (après la transduction de I'ADNc) dans les cellules cultivées en présence d'IGS. Toutes ces données indiquent que l'activation de Notch est un événement récurrent dans les cellules tumorales $I k^{L / L}$ et qui joue un rôle crucial dans le contrôle de leur prolifération.

Deux autres études ont indépendamment révélé une convergence entre lkaros et Notch lors de la leucémogenèse T chez la souris. L. Beverly et A.J. Capobianco [10] ont recherché des mutations capables d'accélérer le développement de leucémies $T$ chez des souris transgéniques exprimant de façon constitutive une forme activée de Notchl, en employant une stratégie de mutagenèse insertionnelle. Ces auteurs ont identifié le locus Ikaros comme le site majeur ciblé par ces insertions, et montré que celles-ci conduisaient à la production de formes

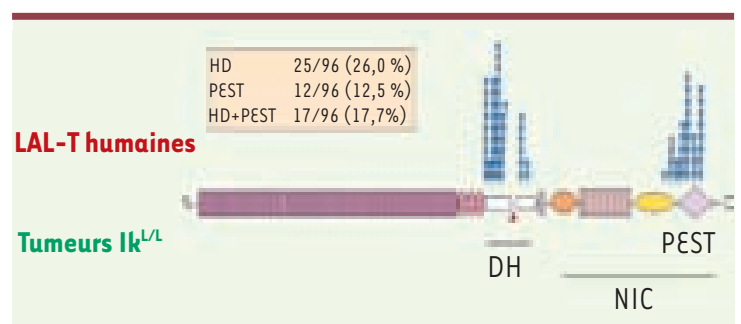

Figure 2. Comparaison entre les mutations ponctuelles de Notch1 trouvées dans les tumeurs $I k^{L / L}$ et les LAL-T chez l'homme [1]. Dans les LAL-T humaines, les mutations se répartissent en deux groupes: domaine d'hétérodimérisation (DH) et région PEST. Chez les souris $I k^{L / L}$, seules des mutations affectant la région PEST ont été trouvées à ce jour. protéiques d'lkaros ayant un rôle de dominant négatif. Par ailleurs, P. LopezNieva et al. [5] ont montré, dans des lymphomes thymiques induits par irradiation, une association fréquente entre mutations ponctuelles d'lkaros et activation de la voie Notch. Ces études et la nôtre pointent donc vers un lien très étroit entre perte de fonction d'lkaros et activation de Notch lors du processus de leucémogenèse T.

\section{Ikaros est un répresseur direct des gènes cibles de Notch}

Quel est le lien entre perte de fonction d'lkaros et activation de la voie Notch? Nos données suggèrent qu'lkaros pourrait être un répresseur direct de l'expression des cibles de Notch. Ainsi, tout comme l'inhibition de Notch par des IGS, la surexpression d'lkaros dans les lignées cellulaires tumorales $\mathrm{I}^{\mathrm{L} / \mathrm{L}}$ conduit à une forte inhibition de leur prolifération, associée à une baisse importante de l'expression de plusieurs gènes cibles de Notch [2]. Par ailleurs, l'analyse de l'expression des cibles de Notch dans des populations de thymocytes pré-tumoraux chez des souris $I k^{L / L}$ très jeunes (âgées de trois semaines) a montré que l'extinction de ces gènes, qui intervient normalement au stade $\operatorname{CD} 4^{+} \operatorname{CD} 8^{+}$de la différenciation thymique, est incomplète chez les mutants. Ainsi, à la fois les données de gain de fonction et de perte de fonction indiquent qu'lkaros pourrait exercer une fonction de répresseur de l'expression de certains gènes cibles de Notch. Une base moléculaire pour cette fonction est suggérée par la similitude entre les séquences d'ADN reconnues par Ikaros et CSL, le facteur transcriptionnel médiateur des effets de Notch ${ }^{1}$. En effet, ces deux protéines reconnaissent toutes les

${ }^{1}$ voir la Figure 1 de la Nouvelle de C. Brou et F. Logeat, p. 685 de ce numéro. deux des séquences contenant le même motif central TGGGAA. In vitro, Ikaros se fixe sur des éléments canoniques reconnus par CSL, ainsi que sur l'élément physiologique du promoteur du gène Hes-1 [2, 10]. Dans des expériences de transactivation, Ikaros inhibe la transcription Notchdépendante à partir de ces éléments. Ainsi Ikaros pourrait réprimer certaines cibles de Notch par compétition avec CSL pour des éléments cibles communs. Une telle fonction permettrait à Ikaros d'assurer une répression dominante de ces gènes cibles qui rendrait ceux-ci insensibles à l'effet des ligands de Notch (Figure 3). Cette fonction pourrait donc expliquer le rôle suppresseur de tumeurs d'lkaros dans les leucémies T murines.

\section{En conclusion}

Les souris $/ k^{L / L}$ offrent un nouveau modèle murin de leucémies T Notch-dépendantes, qui tout comme les LAL-T humaines, sont associées à des mutations secondaires dans Notchl. Ces souris offrent plusieurs perspectives intéressantes: elles permettront d'explorer l'efficacité thérapeutique des IGS, et d'analyser les mécanismes moléculaires qui conduisent à l'activation de Notch dans les leucémies T. Il sera également très intéressant de déterminer si des altérations de la fonction d'lkaros jouent un rôle dans l'activation de la voie Notch dans les leucémies humaines. $\diamond$

Notch activation during

T-cell leukemogenesis

in Ikaros-deficient mice

\section{REMERCIEMENTS}

Nous remercions Robin Jeannet pour la Figure 2. Nos travaux ont bénéficié de subventions de l'ARC, de la Ligue contre le Cancer et de la Fondation de France.

\section{RÉFÉRENCES}

1. Weng AP, Ferrando AA, Lee W, et al. Activating mutations of Notchl in human T cell acute lymphoblastic leukemia. Science 2004 ; 306 : 269-71.

2. Dumortier $A$, Jeannet $R$, Kirstetter $P$, et al. Notch activation is an early and critical event during T-Cell leukemogenesis in Ikaros-deficient mice. Mol Cell Biol $2006 ; 26: 209-20$. 


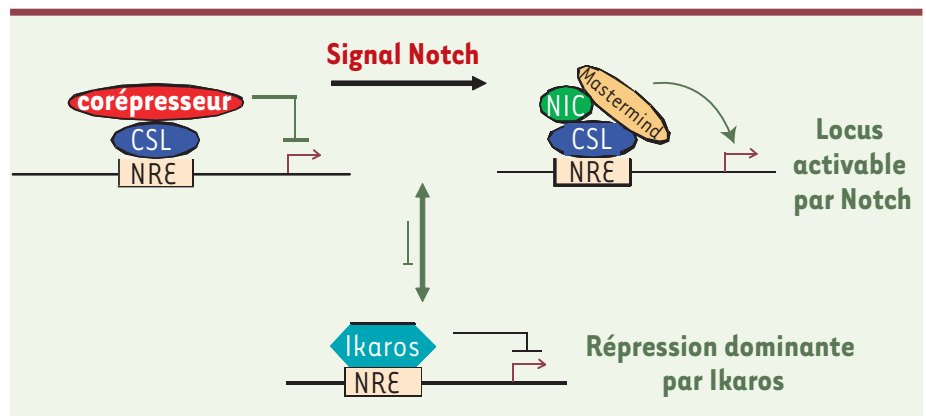

Figure 3. Modèle pour l'action d'lkaros dans la répression de la voie Notch. Dans le modèle classique, le facteur de transcription CSL est fixé aux éléments de réponse de Notch (NRE) en toutes circonstances: en l'absence de signalisation Notch, il interagit avec des corépresseurs pour réprimer la transcription des gènes cibles; en réponse à la signalisation via Notch, une conversion en complexe activateur contenant la partie intracellulaire de Notch (NIC) et la protéine Mastermind se produit. Nous proposons qu'lkaros entre en compétition avec CSL pour la reconnaissance des éléments cibles, et que sa fixation induit une répression dominante.
3. Kirstetter $P$, Thomas M, Dierich A, et al. Ikaros is critical for B cell differentiation and function. Eur J Immunol 2002 ; 32 : 720 - 3.

4. Koipally J, Renold A, Kim J, et al. Repression by Ikaros and Aiolos is mediated through histone deacetylase complexes. EMBO J 1999 ; $18: 3090-100$.

5. Lopez-Nieva P, Santos J, Fernandez-Piqueras J. Defective expression of Notchl and Notch 2 in connection to alterations of c-Myc and lkaros in gamma-radiation-induced mouse thymic lymphomas. Carcinogenesis $2004 ; 25$ : 1299-304.

6. Karlsson A, Soderkvist P, Zhuang SM. Point mutations and deletions in the znfnlal/ikaros gene in chemically induced murine lymphomas. Cancer Res $2002 ; 62: 2650-3$

7. Shimada $Y$, Nishimura M, Kakinuma S, et al. Radiation-associated loss of heterozygosity at the Znfnlal (Ikaros) locus on chromosome 11 in murine thymic lymphomas. Radiat Res 2000 ; 154 : 293-300.

8. Winandy S, Wu P, Georgopoulos K. A dominant mutation in the Ikaros gene leads to rapid development of leukemia and lymphoma. Cell 1995 ; $83: 289-99$.

9. Papathanasiou P, Perkins AC, Cobb BS, et al. Widespread failure of hematolymphoid differentiation caused by a recessive niche-filling allele of the Ikaros transcription factor. Immunity 2003 ; 19 : 131-44.

10. Beverly L, Capobianco AJ. Perturbation of Ikaros isoform selection by MLV integration is a cooperative event in Notch(IC)-induced T cell leukemogenesis. Cancer Cell $2003 ; 3: 551-64$.

\section{NOUVELle}

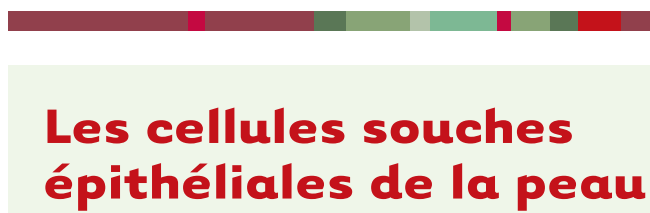

Géraldine Guasch

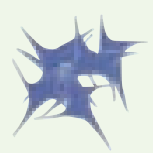

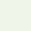

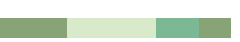

> La peau est la première ligne de défense qui protège le corps de la déshydratation, des blessures et des infections. Les annexes de la peau, comme les poils et les glandes sébacées, ont aussi un effet protecteur. La peau est constituée de deux couches tissulaires: la plus superficielle est l'épiderme et la couche intermédiaire est le derme (Figure IB). Les cellules de l'épiderme, les kératinocytes, sont des cellules spécialisées exprimant une grande variété de filaments intermédiaires appelés kératines, qui confèrent à la peau ses propriétés de résistance mécanique et d'imperméabilité $(\rightarrow)$.

$(\rightarrow) \mathrm{m} / \mathrm{s}$ 2002, $n^{\circ} 1$, p. 45 Chez les mammifères, l'épiderme se renouvelle continuellement. La desquamation des cellules à la surface de la peau doit naturellement être compensée par le renouvellement de l'épiderme, assuré par les kératinocytes de la couche basale qui se divisent activement et se

différencient en cellules de la couche cornée. Ces activités de renouvellement et de réparation de l'épiderme impliquent l'existence de cellules souches (CS).

Les CS sont définies par leur propriété d'autorenouvellement, c'est-à-dire qu'au moins une des cellules filles possèdent les mêmes caractéristiques que la CS initiale, et par leur capacité de différenciation en cellules matures qui composent leur tissu d'origine (Figure IA) $\rightarrow$ ).

Il est généralement admis

$(\rightarrow) \mathrm{m} / \mathrm{s}$ 2004, n 3 , p. 265 que l'épiderme interfolliculaire (EIF) et le follicule pileux comportent leurs propres CS spécialisées capables de maintenir le développement du tissu indépendamment l'un de l'autre.

Contrairement aux CS du follicule pileux, qui sont confinées dans une niche appelée bulge [1] (Figure 1C) $\rightarrow$, les CS de l'ElF résident le long de la membrane basale de l'épiderme. La

Howard Hughes Medical Institute, Laboratory of Mammalian Cell Biology and Development, Rockefeller University, New York, NY 10021, États-Unis. guaschg@rockefeller.edu

couche basale de l'épiderme est composée d'une population hétéro-

$\rightarrow) \mathrm{m} / \mathrm{s}$ 2003, n० 6-7, p. 683 gène de cellules proliférant et se différenciant (Figure IB). La capacité de prolifération et de régénération tissulaire des CS épidermiques a été démontrée par leur culture in vitro suivie par leur transplantation chez les patients atteints de brûlures étendues. Des kératinocytes isolés de l'ElF sont capables de former en culture trois types de clones ayant différents potentiels prolifératifs [2]. Les holoclones, dérivant probablement d'une CS épidermique, représentent les colonies non différenciées ayant le plus fort potentiel de prolifération et pouvant être amplifiés à long terme. Les méroclones, dérivant de cellules en voie de différenciation, ont un potentiel de prolifération intermédiaire et les paraclones, provenant de cellules 